\title{
Bounds for the BMI Eigenvalue Problem ${ }^{\dagger}$
}

\author{
- A Good Lower Bound and A Cheap Upper Bound -
}

\author{
Hisaya FuJIOKA* and Kohta HoshIJIMA*
}

\begin{abstract}
The Bilinear Matrix Inequality (BMI) eigenvalue problem is considered. Upper and Lower bounds of the BMI eigenvalue to combine with the BMI branch and bound algorithm are derived. The proposed lower bound is better in compare to existing one, and still computable via the LMI-optimization. While, the proposed upper bound is computationally cheap utilizing the lower bound optimizer. The worst case gap of the proposed bounds is characterized by the problem data. Numerical examples are also shown.
\end{abstract}

Key Words: BMI eigenvalue problem, branch and bound algorithm, global optimization

\section{Introduction}

Motivated by increasing computer power and sophisticated algorithms ${ }^{14) \sim 16), 20)}$, analysis and design of control systems via numerical optimization is getting widely spread $^{1) \sim 3), 10)}$. Especially, it has been revealed that a number of control problems can be characterized as linear matrix inequalities (LMIs) over the past few years $2), 4), 10$ ). Since LMIs are now easy to handle with computer software ${ }^{4), 19)}$, we can obtain a solution of LMIreducible control problems. However, we sometimes encounter control problems those are at the moment irreducible to LMIs.

Safonov et al. ${ }^{18)}$ pointed out that the bilinear matrix inequality (BMI) is a simple but flexible framework for analysis and synthesis of control systems. It is clear that BMIs can capture a larger class of control problems in compare to LMIs, and hence is potentially a powerful tool. Several researchers have derived BMI-characterizations of their control problems those are not reduced to LMIs yet, and also proposed local ${ }^{5), 8), 9), 11)}$ and global ${ }^{23), 24)}$ algorithms for special classes of BMIs.

Unfortunately, checking the solvability of a BMI is NPhard ${ }^{17)}$. Although this fact indicates that it is computationally difficult to solve BMIs, it does not eliminates the importance of BMIs at all. Goh et al. ${ }^{6), 7)}$ formulated a general class of BMIs as the BMI eigenvalue problem, and investigated its computational issues. Furthermore, a global algorithm ${ }^{6)}$ with upper and lower bounds was proposed based on the branch and bound algorithm.

$\dagger$ Presented at the 40th Annual Conference of ISCIE (1996.5)

* School of Engineering, Osaka University, Suita

(Received July 18, 1996)

(Revised January 29, 1997)
In this paper, we will propose new bounds for the BMI branch and bound algorithm. The proposed lower bound is better in compare to existing one, and still computable via the LMI-optimization. While, the proposed upper bound utilizes the lower bound optimizer, and is computationally cheap. We will also show the worst case gap of proposed bounds. Several numerical examples will be given to show the effectiveness of the BMI branch and bound algorithm with proposed bounds for the BMI eigenvalue problem.

\section{BMI Eigenvalue Problem ${ }^{6)}$}

\section{1 Problem Formulation}

Let $F: \Re^{n_{x}} \times \Re^{n_{y}} \rightarrow \Re^{m \times m}$ be a biaffine function:

$$
F(x, y):=F_{00}+\sum_{i=1}^{n_{x}} x_{i} F_{i 0}+\sum_{j=1}^{n_{y}} y_{j} F_{0 j}+\sum_{i=1}^{n_{x}} \sum_{j=1}^{n_{y}} x_{i} y_{j} F_{i j}
$$

where $F_{i j}=F_{i j}^{T} \in \Re^{m \times m}$ are symmetric matrices. Let also $\mathcal{Q}_{D} \subset \Re^{n_{x}} \times \Re^{n_{y}}$ be a closed bounded hyperrectangle:

$$
\begin{aligned}
\mathcal{Q}_{D}:= & {\left[B_{x_{1}}^{L}, B_{x_{1}}^{U}\right] \times \cdots \times\left[B_{x_{n_{x}}}^{L}, B_{x_{n_{x}}}^{U}\right] } \\
& \times\left[B_{y_{1}}^{L}, B_{y_{1}}^{U}\right] \times \cdots \times\left[B_{y_{n_{y}}}^{L}, B_{y_{n_{y}}}^{U}\right]
\end{aligned}
$$

where $-\infty<B_{x_{i}}^{L} \leq B_{x_{i}}^{U}<\infty,-\infty<B_{y_{j}}^{L} \leq B_{y_{j}}^{U}<\infty$. Now we are ready to formulate the problem:

Definition (BMI Eigenvalue Problem ${ }^{6)}$ ). For given $F$ and $\mathcal{Q}_{D}$, compute

$$
\Phi\left(\mathcal{Q}_{D}\right):=\min _{(x, y) \in \mathcal{Q}_{D}} \bar{\lambda}\{F(x, y)\}
$$

where $\bar{\lambda}\{\cdot\}$ denotes the greatest eigenvalue.

\subsection{BMI Branch and Bound Algorithm}

Goh et al. ${ }^{6}$ ) proposed a branch and bound algorithm for the BMI eigenvalue problem, and derived a condition on which it terminates in finite time:

Lemma 1. ${ }^{6)}$ Given $F$ and $\mathcal{Q}_{D}$, suppose that two functions $\Phi_{L}$ and $\Phi_{U}$ satisfy the following two conditions: 
C1. ${ }^{\forall} \mathcal{Q} \subset \mathcal{Q}_{D}, \Phi_{L}(\mathcal{Q}) \leq \Phi(\mathcal{Q}) \leq \Phi_{U}(\mathcal{Q})$

C2. ${ }^{\forall} \epsilon>0,{ }^{\exists} \delta>0$ such that

$$
{ }^{\forall} \mathcal{Q} \subset \mathcal{Q}_{D}, \quad \operatorname{Size}(\mathcal{Q})<\delta \Rightarrow \Phi_{U}(\mathcal{Q})-\Phi_{L}(\mathcal{Q})<\epsilon
$$

where $\operatorname{Size}(\mathcal{Q})$ denotes the length of the longest side of the hyper-rectangle $\mathcal{Q}$.

Then, $\Phi\left(\mathcal{Q}_{D}\right)$ is obtained in finite time within a given tolerance $\varepsilon>0$ by applying the following algorithm.

Algorithm 1 (BMI Branch and Bound Algorithm ${ }^{6)}$ ). Given $F, \mathcal{P}, \Phi_{L}, \Phi_{U}$, and $\varepsilon>0$.

I. Set $k \leftarrow 0, \mathcal{Q}_{0} \leftarrow \mathcal{Q}_{D}, \mathcal{S}_{0} \leftarrow\left\{\mathcal{Q}_{0}\right\} . L_{0} \leftarrow \Phi_{L}\left(\mathcal{Q}_{0}\right)$, $U_{0} \leftarrow \Phi_{U}\left(\mathcal{Q}_{0}\right)$.

\section{Repeat \{}

R1. Select $\overline{\mathcal{Q}}$ from $\mathcal{S}_{k}$ such that $L_{k}=\Phi_{L}(\overline{\mathcal{Q}})$. $\mathcal{S}_{k+1} \leftarrow \mathcal{S}_{k} \backslash\{\overline{\mathcal{Q}}\}$.

R2. Split $\overline{\mathcal{Q}}$ along its longest edge into $\overline{\mathcal{Q}}_{1}$ and $\overline{\mathcal{Q}}_{2}$.

R3. For $i=1,2$,

$$
\text { If } \Phi_{L}\left(\overline{\mathcal{Q}}_{i}\right) \leq U_{k}, \mathcal{S}_{k+1} \leftarrow \mathcal{S}_{k+1} \cup\left\{\overline{\mathcal{Q}}_{i}\right\} \text {. }
$$

R4. $U_{k+1} \leftarrow \min _{\mathcal{Q} \in \mathcal{S}_{k+1}} \Phi_{U}(\mathcal{Q})$.

R5. Pruning: $\mathcal{S}_{k+1} \leftarrow \mathcal{S}_{k+1} \backslash\left\{\mathcal{Q}: \Phi_{L}(\mathcal{Q})>U_{k+1}\right\}$.

R6. $L_{k+1} \leftarrow \min _{\mathcal{Q} \in \mathcal{S}_{k+1}} \Phi_{L}(\mathcal{Q})$.

R7. $k \leftarrow k+1$.

\}until $U_{k}-L_{k}<\varepsilon$.

Goh et al. ${ }^{6)}$ also proposed functions those can be used as $\Phi_{L}$ and $\Phi_{U}$. In the following sections, we will not modify Algorithm 1, but propose new functions those can be used as $\Phi_{L}$ and $\Phi_{U}$. They will improve the performance of Algorithm 1 in compare to those in Reference 6).

\section{Upper and Lower Bounds}

\section{1 Lower Bound}

In this subsection, we will propose a new function which can be used as a $\Phi_{L}$.

Let us define an affine function $F_{L}$ : $\Re^{n_{x}} \times \Re^{n_{y}} \times$ $\Re^{n_{x} \times n_{y}} \rightarrow \Re^{m \times m}$ related to $F$ by

$$
F_{L}(x, y, W):=F_{00}+\sum_{i=1}^{n_{x}} x_{i} F_{i 0}+\sum_{j=1}^{n_{y}} y_{j} F_{0 j}+\sum_{i=1}^{n_{x}} \sum_{j=1}^{n_{y}} w_{i j} F_{i j}
$$

and consider $\mathcal{Q} \subset \mathcal{Q}_{D}$ of the form

$$
\begin{aligned}
\mathcal{Q}:= & {\left[L_{x_{1}}, U_{x_{1}}\right] \times \cdots \times\left[L_{x_{n_{x}}}, U_{x_{n_{x}}}\right] } \\
& \times\left[L_{y_{1}}, U_{y_{1}}\right] \times \cdots \times\left[L_{y_{n_{y}}}, U_{y_{n_{y}}}\right]
\end{aligned}
$$

Goh et al. ${ }^{6)}$ proposed $\Phi_{L_{G}}$ defined by

$$
\Phi_{L_{G}}(\mathcal{Q}):=\min _{(x, y, W) \in \mathcal{P}_{G}(\mathcal{Q})} \bar{\lambda}\left\{F_{L}(x, y, W)\right\}
$$

to use as $\Phi_{L}$, where

$$
\begin{aligned}
& \mathcal{P}_{G}(\mathcal{Q}):=\mathcal{Q} \times\left\{W \in \Re^{n_{x} \times n_{y}}: w_{i j} \in\left[L_{w_{i j}}, U_{w_{i j}}\right]\right\} \\
& L_{w_{i j}}:=\min \left\{L_{x_{i}} L_{y_{j}}, L_{x_{i}} U_{y_{j}}, U_{x_{i}} L_{y_{j}}, U_{x_{i}} U_{y_{j}}\right\}
\end{aligned}
$$

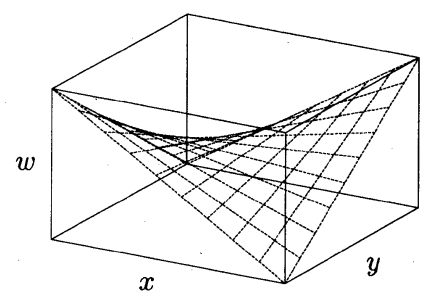

Fig. $1 \quad \mathcal{P}_{G}(\mathcal{Q})$ and $w=x y$

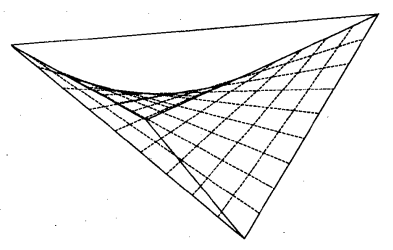

Fig. $2 \mathcal{P}_{H}(\mathcal{Q})$ and $w=x y$

$$
U_{w_{i j}}:=\max \left\{L_{x_{i}} L_{y_{j}}, L_{x_{i}} U_{y_{j}}, U_{x_{i}} L_{y_{j}}, U_{x_{i}} U_{y_{j}}\right\}
$$

Since $F_{L}$ is an affine function and $\mathcal{P}_{G}(\mathcal{Q})$ is an affine set, we can compute $\Phi_{L_{G}}(\mathcal{Q})$ via the LMI-optimization.

Our idea for the reduction of the conservativeness in $\Phi_{L_{G}}$ is fairly simple: just replace $\mathcal{P}_{G}(\mathcal{Q})$ by a smaller affine set $\mathcal{P}_{H}(\mathcal{Q}) \in \Re^{n_{x}} \times \Re^{n_{y}} \times \Re^{n_{x} \times n_{y}}$ defined by

$$
\mathcal{P}_{H}(\mathcal{Q}):=\left\{(x, y, W):\left(x_{i}, y_{j}, w_{i j}\right) \in \operatorname{hull}\left(\mathcal{V}_{i j}\right)\right\}
$$

where hull(.) denotes the convex hull and $\mathcal{V}_{i j} \subset \Re^{3}$ is defined by

$$
\begin{aligned}
\mathcal{V}_{i j}:= & \left\{\left(L_{x_{i}}, L_{y_{j}}, L_{x_{i}} L_{y_{j}}\right),\left(L_{x_{i}}, U_{y_{j}}, L_{x_{i}} U_{y_{j}}\right),\right. \\
& \left.\left(U_{x_{i}}, L_{y_{j}}, U_{x_{i}} L_{y_{j}}\right),\left(U_{x_{i}}, U_{y_{j}}, U_{x_{i}}, U_{y_{j}}\right)\right\}
\end{aligned}
$$

Figs. 1, 2 respectively show $\mathcal{P}_{G}(\mathcal{Q})$ and $\mathcal{P}_{H}(\mathcal{Q})$ for the case $n_{x}=n_{y}=1$ and $\mathcal{Q}=[-0.5,0.5] \times[-0.5,0.5]$. We see that the cuboid $\mathcal{P}_{G}(\mathcal{Q})$ is larger than the tetrahedron $\mathcal{P}_{H}(\mathcal{Q})$ although both of them contain the face of $w=x y$ for $(x, y) \in \mathcal{Q}$. Now we define $\Phi_{L_{H}}$ by

$$
\Phi_{L_{H}}(\mathcal{Q}):=\min _{(x, y, W) \in \mathcal{P}_{H}(\mathcal{Q})} \bar{\lambda}\left\{F_{L}(x, y, W)\right\}
$$

The following result is then obtained:

Property 1. One has

$$
\Phi_{L_{G}}(\mathcal{Q}) \leq \Phi_{L_{H}}(\mathcal{Q}) \leq \Phi(\mathcal{Q})
$$

Proof. It is obvious that we have

$$
\Phi(\mathcal{Q})=\min _{(x, y, W) \in \mathcal{P}(\mathcal{Q})} \bar{\lambda}\left\{F_{L}(x, y, W)\right\}
$$

where $\mathcal{P}(\mathcal{Q})$ is defined by

$$
\mathcal{P}(\mathcal{Q}):=\left\{(x, y, W):(x, y) \in \mathcal{Q}, W=x y^{T}\right\}
$$

For a given $(x, y, W) \in \mathcal{P}(\mathcal{Q})$, we have

$$
\begin{aligned}
& { }^{\forall} i, j,{ }^{\exists} \alpha_{x} \in[0,1], \alpha_{y} \in[0,1], \text { such that } \\
& x_{i}=\alpha_{x} L_{x_{i}}+\left(1-\alpha_{x}\right) U_{x_{i}}, y_{j}=\alpha_{y} L_{y_{j}}+\left(1-\alpha_{y}\right) U_{y_{j}}
\end{aligned}
$$


by definition of $\mathcal{P}(\mathcal{Q})$. Using $\alpha_{x}$ and $\alpha_{y}$, we also have

$$
\begin{aligned}
w_{i j}= & \alpha_{x} \alpha_{y} L_{x_{i}} L_{y_{j}}+\alpha_{x}\left(1-\alpha_{y}\right) L_{x_{i}} U_{y_{j}} \\
& +\left(1-\alpha_{x}\right) \alpha_{y} U_{x_{i}} L_{y_{j}}+\left(1-\alpha_{x}\right)\left(1-\alpha_{y}\right) U_{x_{i}} U_{y_{j}}
\end{aligned}
$$

Noting that $\alpha_{x} \alpha_{y} \in[0,1], \alpha_{x}\left(1-\alpha_{y}\right) \in[0,1],(1-$ $\left.\alpha_{x}\right) \alpha_{y} \in[0,1],\left(1-\alpha_{x}\right)\left(1-\alpha_{y}\right) \in[0,1]$, and

$$
\alpha_{x} \alpha_{y}+\alpha_{x}\left(1-\alpha_{y}\right)+\left(1-\alpha_{x}\right) \alpha_{y}+\left(1-\alpha_{x}\right)\left(1-\alpha_{y}\right)=1
$$

we have $\mathcal{P}(\mathcal{Q}) \subset \mathcal{P}_{H}(\mathcal{Q})$, and the right inequality follows. On the other hand, the left inequality follows the fact $\mathcal{P}_{H}(\mathcal{Q}) \subset \mathcal{P}_{G}(\mathcal{Q})$.

Remark 1. Since hull $\left(\mathcal{V}_{i j}\right)$ is an affine set, $\Phi_{L_{H}}(\mathcal{Q})$ is computable via the LMI-optimization. Actually, $\mathcal{P}_{H}(\mathcal{Q})$ is characterized by $4 n_{x} n_{y}$ (affine and scalar) inequalities, while $\mathcal{P}_{G}(\mathcal{Q})$ by $2\left(n_{x}+n_{y}+n_{x} n_{y}\right)$ inequalities.

Remark 2. Recently, Yada et al. ${ }^{22)}$ also proposed an improved lower bound by replacing $\mathcal{P}_{G}(\mathcal{Q}) . \quad \mathcal{P}_{H}(\mathcal{Q})$ for the case of, e. g., $n_{x}=n_{y}=1$ and $\mathcal{Q}=[0,1] \times[0,1]$ is tighter than their alternative set to $\mathcal{P}_{G}(\mathcal{Q})$. Hence, $\Phi_{L_{H}}$ provides a better lower bound in compare to their one.

Remark 3. The following lower bound of $\Phi(\mathcal{Q})$ is computable via the convex programming:

$$
\Phi_{L_{C}}(\mathcal{Q}):=\min _{(x, y, W) \in \mathcal{P}_{C}(\mathcal{Q})} \bar{\lambda}\left\{F_{L}(x, y, W)\right\}
$$

where $\mathcal{P}_{C}(\mathcal{Q})$ is defined by

$$
\mathcal{P}_{C}(\mathcal{Q}):=\operatorname{hull}\left(\left\{(x, y, w):(x, y) \in \mathcal{Q}, W=x y^{T}\right\}\right)
$$

and we readily have

$$
\Phi_{L_{H}}(\mathcal{Q}) \leq \Phi_{L_{C}}(\mathcal{Q}) \leq \Phi(\mathcal{Q})
$$

However, algorithms applicable to the general convex programming, such as the ellipsoid method, do not achieve good performances in compare to the interior point method in the LMI-optimization.

\section{2 Upper Bound}

It is in fact practical to compute a good upper bound of $\Phi\left(\mathcal{Q}_{D}\right)$. Goh et al. ${ }^{7)}$ proposed the method of centers. Algorithms for special classes of BMIs are found in literature (e. g. 8), 9), 11)).

In this subsection, we also propose an upper bound, which may not be good but computationally cheap: Let us define a function $\Phi_{U_{H}}$ by

$$
\Phi_{U_{H}}(\mathcal{Q}):=\bar{\lambda}\left\{F\left(x^{*}, y^{*}\right)\right\}
$$

where

$$
\left(x^{*}, y^{*}, W^{*}\right):=\arg \min _{(x, y, W) \in \mathcal{P}_{H}(\mathcal{Q})} \bar{\lambda}\left\{F_{L}(x, y, W)\right\}
$$

$\Phi_{U_{H}}(\mathcal{Q})$ is obviously an upper bound of $\Phi(\mathcal{Q})$.

Remark 4. Once we compute $\Phi_{L_{H}}(\mathcal{Q}), \Phi_{U_{H}}(\mathcal{Q})$ is obtained as just the greatest eigenvalue of a fixed matrix $F\left(x^{*}, y^{*}\right)$. This implies that $\Phi_{U_{H}}$ is computationally cheap when combined with $\Phi_{L_{H}}$.

\section{Worst Case Analysis}

In this section, we discuss the worst case gap of proposed bounds.

Let us begin with the following lemma:

Lemma 2. Define a set $\mathcal{V} \subset \Re^{3}$ by

$$
\begin{aligned}
& \mathcal{V}:=\left\{v_{1}, v_{2}, v_{3}, v_{4}\right\} \\
& v_{1}:=\left(x_{1}, y_{1}, x_{1} y_{1}\right), \quad v_{2}:=\left(x_{1}, y_{2}, x_{1} y_{2}\right) \\
& v_{3}:=\left(x_{2}, y_{1}, x_{2} y_{1}\right), \quad v_{4}:=\left(x_{2}, y_{2}, x_{1} y_{2}\right)
\end{aligned}
$$

One has

$$
\max _{(x, y, w) \in \operatorname{hull}_{(\mathcal{V})}}|x y-w| \leq \frac{1}{4}\left|\left(x_{1}-x_{2}\right)\left(y_{1}-y_{2}\right)\right|
$$

Furthermore, the equality holds when

$$
x=\frac{x_{1}+x_{2}}{2}, \quad y=\frac{y_{1}+y_{2}}{2}
$$

Proof. For a given $(x, y, w) \in \operatorname{hull}(\mathcal{V})$, there exists a vector $\alpha \in \mathcal{A} \subset \Re^{4}$ such that

$$
(x, y, w)=\alpha_{1} v_{1}+\alpha_{2} v_{2}+\alpha_{3} v_{3}+\alpha_{4} v_{4}
$$

where $\mathcal{A}$ is defined by

$$
\mathcal{A}:=\left\{\alpha: \alpha_{i} \in[0,1], \alpha_{1}+\alpha_{2}+\alpha_{3}+\alpha_{4}=1\right\}
$$

Then we have

$$
\begin{aligned}
x y-w= & \left(\left(\alpha_{1}+\alpha_{2}\right)\left(\alpha_{2}+\alpha_{3}\right)-\alpha_{1}\right) x_{1} y_{1} \\
& +\left(\left(\alpha_{1}+\alpha_{2}\right)\left(\alpha_{2}+\alpha_{4}\right)-\alpha_{2}\right) x_{1} y_{2} \\
& +\left(\left(\alpha_{3}+\alpha_{4}\right)\left(\alpha_{1}+\alpha_{3}\right)-\alpha_{3}\right) x_{2} y_{1} \\
& +\left(\left(\alpha_{3}+\alpha_{4}\right)\left(\alpha_{2}+\alpha_{4}\right)-\alpha_{4}\right) x_{2} y_{2}
\end{aligned}
$$

From the proof of Property $1,(x, y, x y) \in \operatorname{hull}(\mathcal{V})$, and hence the maximum of $|x y-w|$ is achieved on the surface of $\operatorname{hull}(\mathcal{V})$. Therefore, we substitute $\alpha_{i}=0$ for each $i$ to (9), and obtain the following:

Case 1: $\alpha_{1}=0$ or $\alpha_{4}=0$

$$
\begin{gathered}
|x y-w| \leq \max _{\substack{\alpha_{2} \in[0,1], \alpha_{3} \in[0,1] \\
\alpha_{2}+\alpha_{3} \in[0,1]}} \alpha_{2} \alpha_{3}\left|\left(x_{1}-x_{2}\right)\left(y_{1}-y_{2}\right)\right| \\
\end{gathered}
$$

Case 2: $\alpha_{2}=0$ or $\alpha_{3}=0$

$$
\begin{gathered}
|x y-w| \leq \max _{\alpha_{1} \in[0,1], \alpha_{4} \in[0,1]} \alpha_{1} \alpha_{4}\left|\left(x_{1}-x_{2}\right)\left(y_{1}-y_{2}\right)\right| \\
\alpha_{1}+\alpha_{4} \in[0,1]
\end{gathered}
$$

Hence, the maximum is achieved when

$$
\alpha=\left(0, \frac{1}{2}, \frac{1}{2}, 0\right) \quad \text { or } \quad \alpha=\left(\frac{1}{2}, 0,0, \frac{1}{2}\right)
$$

and (8) follows. This completes the proof.

Now we characterize the worst case gap of proposed bounds by problem data:

Theorem 1. Define $\Delta(\mathcal{Q})$ by

$$
\Delta(\mathcal{Q}):=\Phi_{U_{H}}(\mathcal{Q})-\Phi_{L_{H}}(\mathcal{Q})
$$

Then one has 


$$
\Delta(\mathcal{Q}) \leq \frac{1}{4} \sum_{i=1}^{n_{x}} \sum_{j=1}^{n_{y}} \tilde{\lambda}_{i j}\left(U_{x_{i}}-L_{x_{i}}\right)\left(U_{y_{j}}-L_{y_{j}}\right)
$$

where

$$
\tilde{\lambda}_{i j}:=\max \left\{\bar{\lambda}\left\{F_{i j}\right\}, \bar{\lambda}\left\{-F_{i j}\right\}\right\}
$$

Proof. By definition, we have

$$
\Delta(\mathcal{Q})=\bar{\lambda}\left\{F\left(x^{*}, y^{*}\right)\right\}-\bar{\lambda}\left\{F_{L}\left(x^{*}, y^{*}, W^{*}\right)\right\}
$$

Noting that $\bar{\lambda}(A+B) \leq \bar{\lambda}(A)+\bar{\lambda}(B)$ when both $A$ and $B$ are symmetric,

$$
\begin{aligned}
& \Delta(\mathcal{Q}) \leq \bar{\lambda}\left\{\sum_{i=1}^{n_{x}} \sum_{j=1}^{n_{y}}\left(x_{i}^{*} y_{j}^{*}-w_{i j}^{*}\right) F_{i j}\right\} \\
& \Delta(\mathcal{Q}) \leq \sum_{i=1}^{n_{x}} \sum_{j=1}^{n_{y}} \bar{\lambda}\left\{\left(x_{i}^{*} y_{j}^{*}-w_{i j}^{*}\right) F_{i j}\right\}
\end{aligned}
$$

Invoking Lemma 2, (10) follows.

Remark 5. Theorem 1 implies that the upper bound of $\Delta(\mathcal{Q})$ (RHS of $(10)$ ) becomes its one quarter when

$$
{ }^{\forall} i, j ;\left\{\begin{array}{l}
U_{x_{i}}-L_{x_{i}} \rightarrow \frac{1}{2}\left(U_{x_{i}}-L_{x_{i}}\right) . \\
U_{y_{j}}-L_{y_{j}} \rightarrow \frac{1}{2}\left(U_{y_{j}}-L_{y_{j}}\right)
\end{array}\right.
$$

Hence, it is easy to grid the entire domain to achieve a given tolerance. However, gridding the entire domain may not be practical. Note that $\Delta(\mathcal{Q})$ depends on $\left(x^{*}, y^{*}\right)$ and it may not become its one quarter even in the case of (11).

The next theorem guarantees that Algorithm 1 with $\Phi_{U_{H}}$ and $\Phi_{L_{H}}$ terminates in finite time:

Theorem 2. $\Phi_{U_{H}}$ and $\Phi_{L_{H}}$ fulfill conditions C1 and C2 in Lemma 1.

Proof. From Property 1, it is obvious that $\Phi_{U_{H}}$ and $\Phi_{L_{H}}$ satisfy condition C1. From Theorem 1 and the definition of $\operatorname{Size}(\cdot)$, we have

$$
\Delta(\mathcal{Q}) \leq \frac{1}{4}(\operatorname{Size}(\mathcal{Q}))^{2} \cdot \sum_{i=1}^{n_{x}} \sum_{j=1}^{n_{y}} \tilde{\lambda}_{i, j}
$$

Hence $\Delta(\mathcal{Q})<\varepsilon$ when

$$
\operatorname{Size}(\mathcal{Q})<2 \cdot \sqrt{\frac{\varepsilon}{\sum_{i=1}^{n_{x}} \sum_{j=1}^{n_{y}} \tilde{\lambda}_{i, j}}}
$$

This completes the proof.

\section{Numerical Examples}

In this section, we demonstrate the effectiveness of the BMI branch and bound algorithm with proposed bounds. The algorithm is implemented on $\mathrm{Xmath}^{21)}$ combined with SP ${ }^{19)}$.

\section{1 Demonstrative Example}

In this subsection, we consider a $\mathrm{BMI}^{6)}$ defined by

$$
F(x, y)=F_{00}+F_{10} x+F_{01} y+F_{11} x y
$$

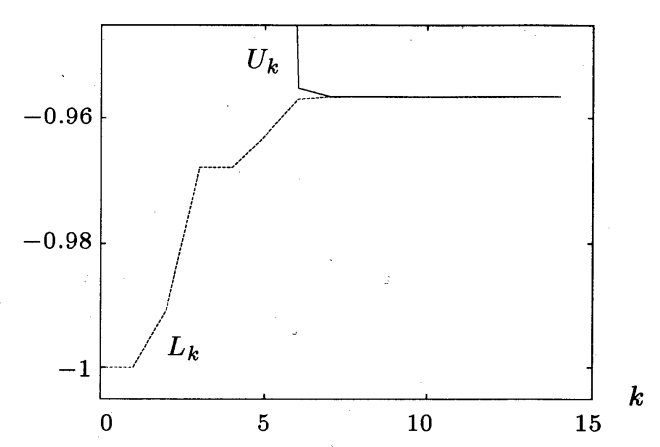

Fig. 3 Progress of Upper $\left(U_{k}\right)$ and Lower $\left(L_{k}\right)$ Bounds

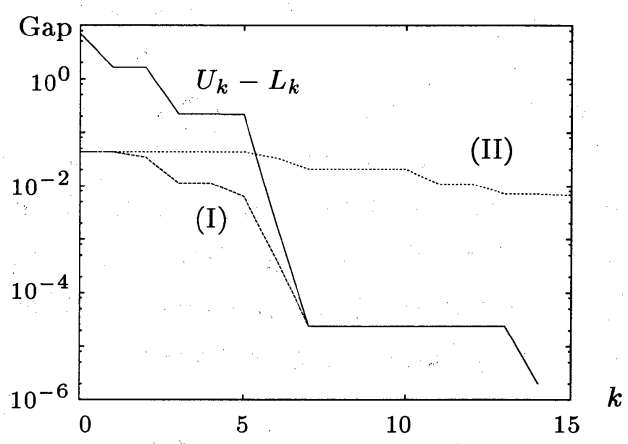

Fig. 4 Comparisons of Performances of Bounds

$$
\left[\begin{array}{ll}
F_{00} & F_{10} \\
F_{01} & F_{11}
\end{array}\right]=\left[\begin{array}{ccc:ccc}
-10 & -0.5 & -2 & 9 & 0.5 & 0 \\
-0.5 & 4.5 & 0 & 0.5 & 0 & -3 \\
-2 & 0 & 0 & 0 & -3 & -1 \\
\hdashline-1.8 & -0.1 & -0.4 & 0 & 0 & -2 \\
-0.1 & 1.2 & -1 & 0 & -5.5 & 3 \\
-0.4 & -1 & 0 & 2 & 3 & 0
\end{array}\right]
$$

and $\mathcal{Q}_{D}=[-0.5,2] \times[-3,7]$.

We apply the BMI branch and bound algorithm with proposed bounds and $\varepsilon=10^{-5}$ to the problem. Fig. 3 shows the progress of the algorithm with proposed bounds. After 14 iterations, (gap) $<10^{-5}$ is satisfied. It should be noted that the pruning step in the algorithm works well for this problem. Actually, only 2 branches appear simultaneously, i. e., $\mathcal{S}_{k}$ has 2 elements at most, until the tolerance is satisfied.

To evaluate the performance of $\Phi_{U_{H}}$, we apply Algorithm 1 replacing $U_{k}$ 's by the optimal value. Lower bounds $L_{k}$ 's are computed using $\Phi_{L_{H}}$. The results are plotted in Fig. 4: The curves labeled by $U_{k}-L_{k}$ and (I) indicate the gap of bounds related to $\Phi_{U_{H}}$ and $\Phi_{L_{H}}$, and that between lower bounds and the optimal value, respectively. We see that the same accuracy is obtained when $k \geq 7$, and hence, in this case, we may conclude that a cheap upper bound $\Phi_{U_{H}}$ is sufficiently good.

Next, let us compare the performances of the lower bounds related to $\Phi_{L_{H}}$ and $\Phi_{L_{G}}$. For the purpose, we apply Algorithm 1 with $U_{0}=$ (the optimal value) and $\Phi_{L}=\Phi_{L_{G}}$. The result is plotted as (II) in Fig. 4. With lower bounds derived by $\Phi_{L_{G}}$, the tolerance $\varepsilon=10^{-3}$ is 


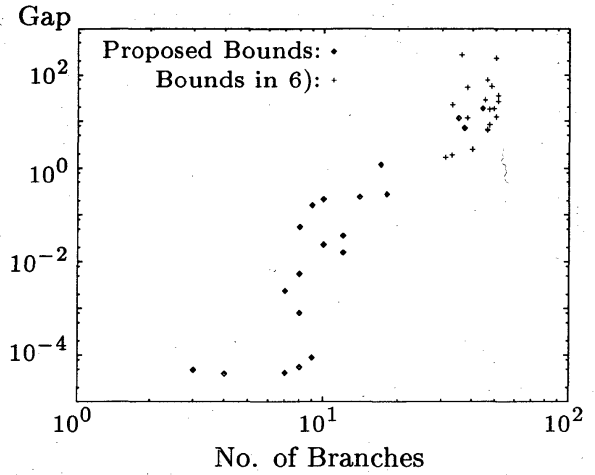

Fig. 5 Error vs. No. of Branches

not satisfied after 15 iterations even if we employ the optimal value as an upper bound. Actually, in the case, we need more than 800 iterations and more than 600 branches to satisfy $\varepsilon=10^{-5}$. Note that the difference of curves (I) and (II) comes from that of lower bounds. The results implies that $\Phi_{L_{H}}$ fairly improves the performance of Algorithm 1.

\subsection{Randomly Generated BMI Problems}

In this subsection, we compare proposed bounds and existing ones ${ }^{6)}$ by solving randomly generated BMIs.

We have generated more than 100 BMIs for $m=3,4,5$, $n_{\dot{x}}<10, n_{y}<10$. With proposed bounds, (gap) $<10^{-5}$ was achieved by applying Algorithm 1 until $k=50$ for about $70 \%$ problems, while it was not achieved with existing bounds ${ }^{6)}$.

For the $30 \%$ harder problems, pairs of the gap and the number of branches at $k=50$ are plotted in Fig. 5 . We see that smaller gap is achieved with less number of branches with proposed bounds.

\section{3 Stabilization of Double Integrator}

Fixed order controller design is a typical application of the BMI framework. In this subsection, we consider the problem to find a first order controller which stabilizes a double integrator.

The problem is easily formulated as a BMI: Find $X \in$ $\Re^{3 \times 3}, X=X^{T}>0$ and $K \in \Re^{2 \times 3}$ satisfying

$$
(A+B K C)^{T} X+X(A+B K C)<0
$$

where $A, B$ and $C$ are defined by

$$
\left[\begin{array}{ll}
A & B \\
C & 0
\end{array}\right]:=\left[\begin{array}{ccc:cc}
0 & 1 & 0 & 0 & 0 \\
0 & 0 & 0 & 1 & 0 \\
0 & 0 & 0 & 0 & 1 \\
\hdashline 1 & 0 & 0 & 0 & 0 \\
0 & 0 & 1 & 0 & 0
\end{array}\right]
$$

Iwasaki and Skelton pointed out that the $X Y$-centering algorithm ${ }^{11)}$, a type of local optimization algorithm, works well for the problem. Applying the $X Y$-centering

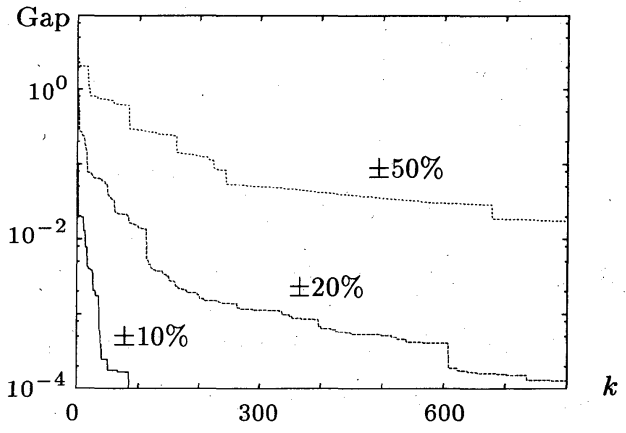

Fig. 6 Progress of Gap Varying Domain

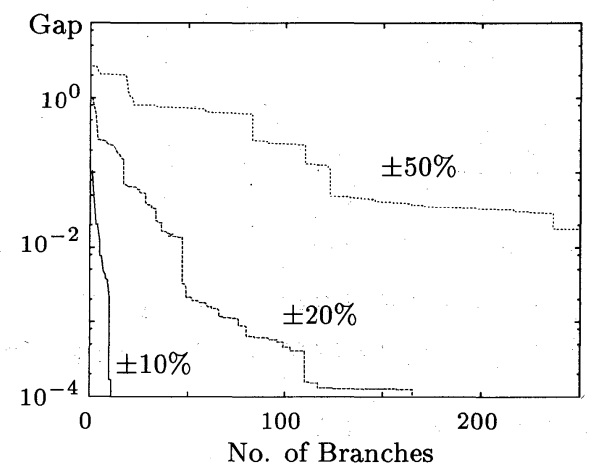

Fig. 7 Error vs. No. of Branches Varying Domain

algorithm, we actually obtain a solution of (12):

$$
\begin{aligned}
& X=X_{0}:=\left[\begin{array}{ccc}
1.1458 & -0.6910 & -1.6325 \\
-0.6910 & 1.7966 & 2.0712 \\
-1.6325 & 2.0712 & 3.6253
\end{array}\right] \\
& K=K_{0}:=\left[\begin{array}{cc}
-2.5695 & 3.5950 \\
1.2955 & -2.1061
\end{array}\right]
\end{aligned}
$$

where $X_{0}$ is found in 11). With a fixed $X=X_{0}, K_{0}$ is obtained by solving (12) as an LMI in $K$.

Now we solve the BMI (12) as a BMI eigenvalue problem, i. e., we will optimize the greatest eigenvalue of the left hand side of (12), where the domain of $X$ and $K$ will be set around (13) and (14). Note that the pair of (13) and (14) is not the optimizer although it is a feasible solution of (12).

The results are shown in Figs. 6, 7, where the domain is determined by, e. g., $X \in\left[0.9 X_{0}, 1.1 X_{0}\right]$ and $K \in\left[0.9 K_{0}, 1.1 K_{0}\right]$ (element wise) for the case of $\pm 10 \%$. When the domain is the case of $\pm 10 \%$, we need less than 100 steps and less than 15 branches to achieve (gap) $<10^{-4}$. While, if the domain is the case of $\pm 50 \%$, the gap is not less than $10^{-2}$ even at $k=800$ having more than 200 branches.

Through this example, we see that the BMI problem becomes much harder when its domain gets larger. 


\section{Conclusions}

We have proposed upper and lower bounds for the BMI eigenvalue problem. The proposed lower bound is computable via the LMI-optimization, and better than existing one ${ }^{6}$ by considering the geometry of the problem. The proposed upper bound utilizes the lower bound optimizer, and is computationally cheap. We also have derived the worst case gap of proposed bounds.

Through numerical examples, we have compared proposed bounds and existing ones ${ }^{6}$, and have shown the effectiveness of the BMI branch and bound algorithm combined with proposed bounds.

Although proposed bounds improve the performance of the BMI branch and bound algorithm, it is not sufficiently good especially when its domain is large, as pointed out in Section 5. Hence, further enhancement is still required. An approach with parallel computation ${ }^{13)}$ and a new branching scheme ${ }^{12)}$ have been reported very recently. Better and cheaper bounds may found for a certain control problem.

Finally, the authors wish to express their appreciations to Dr. Goh, Prof. Safonov, and Prof. Hara for their valuable comments. Thanks also to one of the anonymous referees for pointing out the gap between $\mathcal{P}_{H}$ and $\mathcal{P}_{C}$.

\section{References}

1) S. P. Boyd and C. H. Barratt: Linear Controller Design: Limits of Performance, Prentice Hall (1991).

2) S. Boyd, L. E. Ghauli, E. Feron and V. Balakrishnan: Linear Matrix Inequalities in Systems and Control Theory, SIAM books (1994).

3) M. A. Dahleh and I. J. Diaz-Bobillo: Control of Uncertain Systems: A Linear Programming Approach, Prentice Hall (1995).

4) P. Gahinet, A. Nemirovski, A. J. Laub and M. Chilali: LMI Control Toolbox, The Math Works, Inc. (1995).

5) K. C. Goh, J. H. Ly, L. Turan and M. G. Safonov: " $\mu / K_{m-}$ Synthesis via Bilinear Matrix Inequalities," in Proc. of the 33rd Conf. Decision Control, 2032-2037 (1994)

6) K. C. Goh, M. G. Safonov and G. P. Papavassilopoulos: "A Global Optimization Approach for the BMI Problem," in Proc. of the 33rd Conf. Decision Control, 2009-2014 (1994).

7) K. C. Goh, L. Turan, M. G. Safonov, G. P. Papavassilopoulos and J. H. Ly: "Biaffine Matrix Inequality Properties and Computational Methods," in Proc. Amer. Contr. Conf., 850-855 (1994).

8) K. M. Grigoriadis and R. E. Skelton: "Low-Order Control Design for LMI Problems Using Alternating Projection Methods," Automatica, vol. 32, no. 8, 1117-1125 (1996)

9) M. Ikeda, G. Zhai and Y. Fujisaki: "Decentralized $\mathcal{H}_{\infty}$ Controller Design for Large-Scale Systems: A Matrix Inequality Approach Using a Homotopy Method," in Proc. of the 35th Conf. Decision Control, 1-6 (1996).

10) T. Iwasaki: "A Unified Matrix Inequality Approach to Linear Control Design," Ph. D. dissertation, Purdue Univer- sity (1993).

11) T. Iwasaki and R. E. Skelton: "The $X Y$-Centering Algorithm for the Dual LMI Problem: A New Approach to Fixed-Order Control Design," International Journal of Control, vol. 62, no. 6, 1257-1272 (1995).

12) M. Kawanishi and T. Sugie: "BMI Global Optimization via BB Method Considering Bilinearity," in Proc. of the 19th SICE Symposium on Dynamical System Theory, 5962 (1996), In Japanese.

13) S. Liu and G. P. Papavassilopoulos: "Numerical Experience with Parallel Algorithms for Solving the BMI Problem," in Proc. of the 13th World Congress of IFAC, vol. D, 387-391 (1996).

14) A. Nemirovski and P. Gahinet: "The Projective Method for Solving Linear Matrix Inequalities," in Proc. Amer. Contr. Conf., 840-844 (1994).

15) Y. Nesterov and A. Nemirovski: Interior Point Polynomial Methods in Convex Programming: Theory and Applications, SIAM books (1994).

16) R. Saigal: Linear Programming, Kluwer Academic Publishers (1995).

17) O. Toker and H. Özbay: "On the $\mathcal{N} \mathcal{P}$-Hardness of Solving Bilinear Matrix Inequalities and Simultaneous Stabilization with Static Output Feedback," in Proc. Amer. Contr. Conf., 2525-2526 (1995).

18) M. G. Safonov, K. C. Goh and J. H. Ly: "Control System Synthesis via Bilinear Matrix Inequalities," in Proc. Amer. Contr. Conf., 45-49 (1994).

19) L. Vandenberghe and S. Boyd: SP User's Guide, on-line document (1994).

20) L. Vandenberghe and S. Boyd: "Semidefinite Programming," SIAM Review, vol. 38, no. 1, 49-95 (1996).

21) Xmath Basics, Integrated Systems, Inc. (1996).

22) T. Yada, M. Kawanishi and T. Sugie: "Controller Design of 2 Mass-Spring System via BMI," in Proc. of the 40th Annual Conference of ISCIE, 451-452 (1996), In Japanese.

23) Y. Yamada and S. Hara: "A Global Algorithm for Scaled Spectral Norm Optimization," in Proc. of the 25th SICE Symposium on Control Theory, 177-182 (1996).

24) Y. Yamada, S. Hara and H. Fujioka: "Global Optimization for Constantly Scaled $\mathcal{H}_{\infty}$ Control Problem," in Proc. Amer. Contr. Conf., 427-430 (1995).

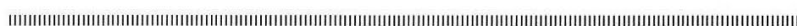

\section{Hisaya Fujıoka (Member)}

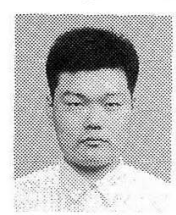

He received the B. S., M. S., and Ph. D. degrees in engineering from Tokyo Institute of Technology, Tokyo, Japan, in 1990, 1992, and 1995 , respectively. He is currently a Research Associate at School of Engineering, Osaka University. His current research interests include sampled-data control, robust control, and computer aided control system design.

\section{Kohta Hoshisima (Student Member)}

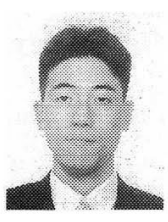

$\mathrm{He}$ received the $\mathrm{B}$. S. degree in engineering from Osaka University, Suita, Osaka, Japan, in 1996. He is currently a student at Master course, School of Engineering, Osaka University. His research interests include control systems design via BMIs. 